\title{
Lysosomal Cholesterol Accumulation in Model Atherosclerotic Foam Cells
}

\author{
Brian E. Cox, Evelyn E. Griffin, J. Erin Tillman and W. Gray (Jay) Jerome
}

Vanderbilt University Medical Center, Nashville TN 37232

Atherosclerosis (hardening of the arteries) remains a major cause of death in the United States. Massive cholesterol accumulation within macrophage lysosomes is a key event in atherosclerosis. The accumulation produces "foamy" appearing cells. The mechanism for this foam cell formation is unknown. In previous studies we showed that cultured macrophages incubated with oxidized low density lipoprotein (oxLDL) show a similar lysosomal cholesterol accumulation. This accumulation occurred in two stages. At first, the cholesteryl esters (CE) in the oxLDL were hydrolyzed to free cholesterol but the resultant cholesterol was unable to leave the lysosome. Following this, hydrolysis of oxLDL CE was inhibited resulting in lysosomes that contained significant free and esterified cholesterol. In contrast, incubation of cells with acetylated LDL (acLDL) did not produce significant lysosomal cholesterol accumulation. The cells stored equivalent amounts of cholesterol to those treated with oxLDL but the cholesterol was stored as cytoplasmic cholesteryl ester droplets. This indicates that CE from acLDL was hydrolyzed normally in lysosomes and the resulting free cholesterol was delivered to the cytoplasm for reesterification to CE.

As a follow up to these studies we have investigated other potentially atherogenic lipid particles for their ability to induce lysosomal cholesterol accumulation. THP-1 macrophages in culture were incubated with aggregated lipoproteins. The aggregates are internalized by unregulated pathways and delivered to lysosomes. When macrophages were incubated with aggregates of LDL, oxLDL, or acLDL, free cholesterol more than doubled and esterified cholesterol was increased more than 3 fold. Morphological studies showed that the excess cholesterol was primarily found within the hydrolytic compartment (late endosome/lysosome) as evidenced by co-localization of cholesterol staining (filipin) and LAMP-1 staining.

In addition to lipoproteins, the extracellular milieu in atherosclerotic lesions is rich in various forms of lipid particles [1-3]. We have shown that cholesterol-rich artificial particles of similar composition to those found in lesions can promote lysosomal cholesterol loading in macrophages [4]. These particles are useful for delivering defined lipid mixtures to lysosomes. Incubation of macrophages with cholesterol-enriched artificial lipid particles produced lysosomal engorgement and free and esterified cholesterol augmentation similar to oxLDL treatment. Figure 1 shows that, as with aggregated lipoproteins, filipin staining for unesterified cholesterol co-localized with LAMP-1 staining indicating localization of cholesterol accumulation within a hydrolytically active compartment (lysosome/late endosome).

These data indicate a failure of lysosomal CE hydrolysis as a major factor in lysosomal lipid accumulation. The cause of this inhibition is not known. However, our recent work indicates that the lipid-filled lysosomes (AcPase and LAMP1 positive) are not acidified. Lysosensor Yellow/Blue has yellow fluorescence in acid but at neutrality it emits blue fluorescence. Cells loaded for two days or less with ox-LDL have distinctly acidic lipid-engorged lysosome. AcLDL-treated cells continued to show acidic lysosomes up to 6 days. In contrast, oxLDL-treated cells show few if any acidic lysosomes after 3 days even though nile red (lipid stain) and LAMP-1 staining continued to 
indicate the presence of lipid filled lysosomes. The shift occurring between days 2 and 3 coincides with the inhibition of CE hydrolysis.

These observations are consistent with the hypothesis that, with heavy lipoprotein loading, FC accumulation in lysosomes irreversibly inhibits lysosomal function leading to an almost complete blockage of $\mathrm{FC}$ and $\mathrm{CE}$ clearance from lysosomes resulting in cellular dysfunction. Thus, this aspect of foam cell formation can be viewed as a modified form of lysosomal storage disease.

\section{References}

[1] H Kruth, Am J Pathol 114 (1984) 201.

[2] JR Guyton et al., Eur Heart J 11 (Suppl E) (1990) 20.

[3] E Smith, et al., J Atheroscler Res 8 (1968) 399.

[4] W G Jerome and P G Yancey, Microsc Microanal 9 (2003) 54.

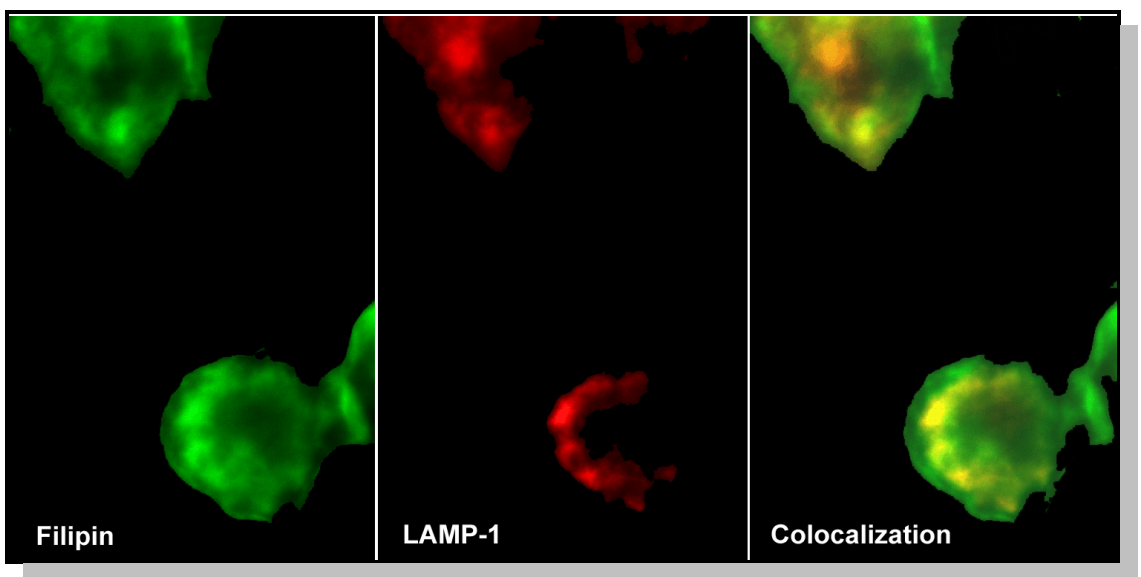

Figure 1. THP-1 cells stained to demonstrate the presence of free cholesterol (Filipin) and immunostained to localize LAMP-1. The yellow areas in the right hand panel indicate that significant free cholesterol is contained within LAMP-1 positive vesicles. 